\title{
Metastatic Staphylococcus aureus endophthalmitis: a case report
}

\author{
C J O'BRIEN AND G M KYLE \\ From the Department of Ophthalmology, King's College Hospital, London
}

SUMmary A case of bilateral metastatic Staphylococcus aureus endophthalmitis is presented. It evolved over a two-week period, causing some diagnostic confusion. Treatment with fusidic acid and benzylpenicillin resulted in the retention of useful vision in one eye and good vision in the other.

Staphylococcal endophthalmitis is a rare condition, usually a complication of intraocular surgery or penetrating injury, but it may also be due to metastatic spread from a distant source.' Generally considered to run a rapid course, it may result in severe damage to or loss of the eye. The poor prognosis is related in part to the delay in diagnosis, inadequate treatment, and the virulence of the organism. Patients infected with Staph. epidermidis tend to fare better than those infected with Staph. aureus, in whom up to $66 \%$ lose useful vision. In a review of 70 cases of endophthalmitis nine were reported to be of Staph. aureus infection; all of these had had previous ocular surgery and only two of them retained useful vision. $^{2}$

Bilateral metastatic staphylococcal involvement is rare. ${ }^{3}$ De Wolff Rouendal described a clinicopathological case of bilateral endophthalmitis in a patient with Staph. aureus septicaemia, who subsequently died as a result of overwhelming infection. Postmortem examination showed septic retinitis with bilateral central retinal vein occlusion.

We report below a case of bilateral endophthalmitis in a patient with Staph. aureus septicaemia, in which the clinical picture and the slow evolution of the endophthalmitis caused some confusion with fungal endophthalmitis as the clinical diagnosis. So far as we are aware this is the first case in which the progression of a Staph. aureus focus in the fundus has been described.

\section{Case report}

A 54-year-old man who was a poorly controlled Correspondence to Mr G M Kyle, Wrexham Maelor Hospital, Croesnewydd Road, Wrexham, Clwyd LL13 7TD. diabetic was admitted to hospital with a three-week history of fatigue, general malaise, and weight loss. He was known to have ischaemic heart disease, peripheral vascular disease, peripheral neuropathy, and background diabetic retinopathy. Physical examination showed a pale, thin man; temperature $36^{\circ} \mathrm{C}$. He had a right axillary pleural rub and bilateral coarse crepitations. Chest $x$-ray showed right lower lobe consolidation. The initial diagnosis was that of a chest infection superimposed on congestive cardiac failure.

He was seen in the Eye Department on the day of admission with a 10-day history of floaters and loss of vision in his right eye only. The visual acuity was counting fingers in the right eye and 6/12 in the left eye. The anterior segments were unremarkable. There were cells in the posterior vitreous in the right eye, and fundal examination revealed a large white chorioretinal lesion at the right macula (Fig. 1). There were four satellite lesions adjacent to the inferonasal border of the main lesion. Three of these appeared to be in or on the retina, but one was growing forward into the vitreous. The left fundus was normal apart from a tiny yellow lesion at the fovea. This was taken to be a diabetic exudate.

The initial impression of the lesion in the right eye suggested a fungal aetiology, and no treatment was begun while the results of blood cultures were awaited.

The patient was examined again two days later. He now had a right afferent pupillary defect. There was a cellular reaction in the anterior chambers and vitreous of both eyes. Fundal examination showed an increase in size of the previously noted satellite lesions and two new, small, yellow dot lesions in relation to the inferior temporal vessels. There was 
Fig. 1 Metastatic Staph. aureus endophthalmitis: chorioretinal focus at the right macula with satellite lesions seen at lower edge.

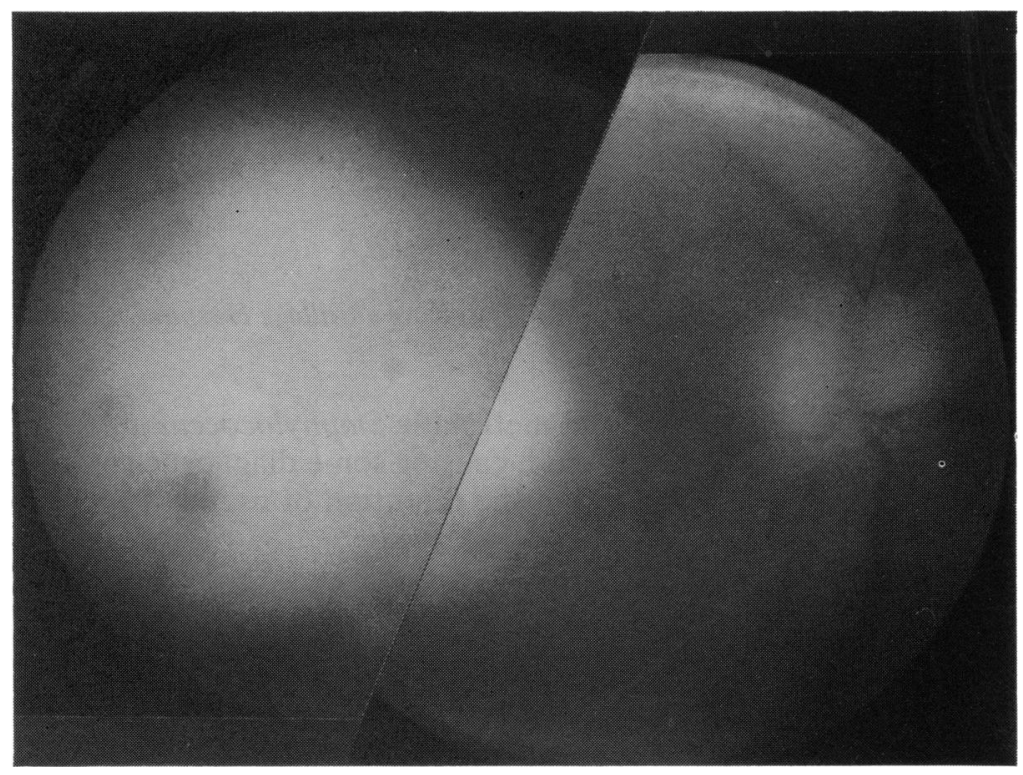

a semicircular preretinal white lesion below the macular lesion. This was presumed to be a gravitational collection of white cells. The left fundus was unchanged.

It was now known that Staph. aureus had been grown from four out of four blood cultures and from the urine. No hyphae were seen in the blood or urine. He was started on fusidic acid $500 \mathrm{mg}$ three times a day orally, and benzylpenicillin 2 megaunits four times a day intravenously. He was also started on oral ketoconazole $200 \mathrm{mg}$ twice daily and oral 5 flucytosine $1.5 \mathrm{~g}$ four times a day, ${ }^{4}$ but it was decided that a diagnostic vitrectomy should be performed on the right eye because there was now evidence of progression in the right eye plus involvement of the left eye.

A vitrectomy with the Ocutome through the pars plana was performed under general anaesthetic two days later. As much vitreous as possible was removed. The fluffly satellite lesion which was growing forward from the main chorioretinal lesion was seen to be removed by the cutter. Microscopic examination of the aspirate revealed Gram-positive cocci and no hyphae. There was no growth from the vitreous. The vitreous drug levels were: 5 flucytosine, $1 \mu \mathrm{g} / \mathrm{ml}$; ketoconazole, not detected. The antifungal treatment was stopped. The systemic antibiotics were continued and the patient started on gentamicin $1 \%$ eyedrops four times a day and atropine eyedrops $1 \%$ twice daily to the right eye.

The intraocular inflammation subsided completely over the next three weeks, and the visual acuity was counting fingers in the right eye and 6/9 in the left eye. Fundal examination at seven months showed a large atrophic chorioretinal scar at the right macula (Fig. 2).

The source of the septicaemia was never established. An intravenous pyelogram, abdominal ultrasound, echocardiogram, gallium scan, and bone scan were all unhelpful. Clinically it was thought that a renal focus was the most likely source.

\section{Discussion}

Although it was not possible to grow Staph. aureus



Fig. 2 Atrophic chorioretinal scar at the right macula seven months postoperatively. 
from the vitreous, infection with this organism would seem the most likely diagnosis. The patient had been on antibiotics to which Staph. aureus was known to be sensitive for two days prior to the vitrectomy, so it is understandable that although Gram-positive cocci were seen no organism was cultured.

The cellular reaction in the left eye is less obviously related to metastatic involvement from staphylococcal septicaemia, but no other cause was apparent. There is an interesting case report of a unilateral iridocyclitis for which no cause was found until a swelling of the ciliary body developed after one month. ${ }^{5}$ This was subsequently found to be a metastatic abscess of the ciliary body and Staph. aureus was isolated. A similar mechanism may explain the anterior uveitis in the left eye in our case, with the natural history being curtailed by the systemic antibiotic therapy.

The site of initial ocular infection in the right eye must be assumed to be the choroid, as the infection was blood-borne. In keeping with this is the observation of the development of a relative afferent pupillary defect two days after initial ophthalmic examination. As there was no funduscopic involvement of the optic nerve head, this development would imply increasing involvement of the neuroretina. The size of the main macular lesion had not significantly increased during this time, so it must be assumed that the inflammatory process was progressing anteriorly.

The case referred to above ${ }^{5}$ and our own case demonstrate that Staph. aureus endophthalmitis can run a fairly slow course. It was this which gave the initial clinical impression of fungal endophthalmitis, for it was thought that a bacterial endophthalmitis (as the blood cultures suggested) would have progressed much more rapidly in the 12 days between first symptoms and institution of specific therapy.

Septicaemia in a possibly immunocompromised patient does not preclude a diagnosis of fungal endophthalmitis, so the need for a diagnostic vitrectomy is clear. ${ }^{2}$

The response to treatment was satisfactory in that the damage to the right eye was contained, and the left eye settled without obvious sequelae. This case demonstrates that it is possible to treat staphylococcal endophthalmitis without recourse to intravitreal antibiotics. There is still some doubt about possible retinal toxicity of intraocular gentamicin, ${ }^{6}$ and, as the initial clinical differentiation between fungal and bacterial infection may be misleading, there are grounds for caution in their use. Fusidic acid seems a particularly appropriate systemic antibiotic in view of its excellent ocular penetration..$^{78}$ It is found in effective concentration in the aqueous of quiet eyes, and in inflamed eyes penetration is increased, with, significantly, vitreous levels two or three times greater than the aqueous concentrations. If the main site of ocular infection is in the uveal tract, this may explain such a good response to systemic antibiotics.

Possibly the best route for treatment is different for metastatic endophthalmitis than for postsurgical endophthalmitis. The maximum bacterial concentration is likely to be at the site of initial infection, and so systemic antibiotics are more likely to reach the nidus of infection in metastatic endophthalmitis.

It is interesting to note that Ketoconazole was not detected in the vitreous despite the patient having been on treatment for 48 hours prior to surgery. It should be pointed out, however, that the last dose of ketoconazole was given 14 hours prior to vitrectomy. Unfortunately blood levels were not measured at the same time, so we cannot be categorical about the poor vitreous penetration, but our finding is in agreement with that of Stern et al. , ${ }^{9}$ who found only a trace of ketoconazole in the vitreous of their patient six hours after an $800 \mathrm{mg}$ oral dose. Experimental work in rabbits also demonstrates poor vitreous penetration by ketoconazole. ${ }^{10}$

We thank Mr E W G Davies for permission to report this case under his care, Mr C Clements for help with the illustrations, and Miss C Sparshott and Miss B Conlon for their secretarial assistance.

\section{References}

1 Farber BP, Weinbaum DL, Dummer JS. Metastatic bacterial endophthalmitis. Arch Intern Med 1985; 145: 62-4.

2 Rowsey JJ, Newson DL, Sexton DJ, Horms WK. Endophthalmitis: current approaches. Ophthalmology 1982; 89: 1055-66.

3 De Wolff-Rouendal D. Bilateral metastatic endophthalmitis caused by Staphylococcus aureus: a clinopathological case history. Doc Ophthalmol 1979; 48: 319-26.

4 Salmon JF, Partridge BM, Spalton DJ. Candida endophthalmitis in a heroin addict: a case report. Br J Ophthalmol 1983; 67: 306-9.

5 Gamel JW, Allansmith MR. Metastatic staphylococcal endophthalmitis presenting as chronic iridocyclitis. Am J Ophthalmol 1974; 77: 454-8.

6 Conway BP, Campochisco PA. Macular infarction after endophthalmitis treated with vitrectomy and intravitreal Gentacin. Arch Ophthalmol 1986; 104: 367-77.

7 Chadwick AJ, Jackson B. Intraocular penetration of the antibiotic fucidin. Br J Ophthalmol 1969; 53: 26-9.

8 Williamson J, Russell F, Doig WM, Paterson RWW. Estimation of sodium fusidate levels in human serum, aqueous humour, and vitreous body. Br J Ophthalmol 1970; 54: 126-30.

9 Stern WH, Tamura E, Jacobs RA, et al. Epidemic postsurgical Candida parapsilosis endophthalmitis. Ophthalmology 1985; 92: 1701-9.

$10 \mathrm{Chu}$ W, Foster CS, Moran K, Giovanoni R. Intraocular penetration of ketoconazole in rabbits. ARVO abstracts: Invest Ophthalmol Vis Sci (suppl). St Louis: Mosby, 1979: 133.

Accepted for publication 7 January 1987. 